\title{
Psychosocial Stress and its Effect on Periodontal Tissues using Malondialdehyde as Oxidative Stress Biomarker
}

\author{
Dr. Anuradha Mokashi, ${ }^{1}$ Dr. Keshava Abbayya, ${ }^{1}$ Dr. Siddhartha Varma, ${ }^{1}$ Dr. Sameer Zope, ${ }^{1}$ \\ Dr. Girish Sugarimath, ${ }^{1}$ Dr. Apurva Pisal ${ }^{1}$ \\ ${ }^{1}$ Department of Periodontology, School of Dental Sciences, Krishna Institute of Medical Sciences, \\ Deemed University, Karad, Maharashtra, India.
}

\begin{abstract}
Background: Stress is playing a major role as an aetiologic factor in the initiation of many systemic diseases. Similarly, it is also thought to be an aetiologic factor in the progression of periodontal diseases.

Aim: The production of free radical species is associated with various inflammatory diseases and also during stressful conditions. Hence, an effort in the present study is done to correlate psychosocial stress and periodontitis using serum MDA as an oxidative stress biomarker.

Materials and Methods: A current cross-sectional study included 201 individuals between 20 to 60 years of age. A periodontal examination included Probing Pocket Depth, CAL, Plaque Index, and Gingival Index. According to CAL individuals were divided into four groups i.e. healthy, slight $(\mathrm{CAL}=1-2 \mathrm{~mm})$, moderate $(\mathrm{CAL}=3-4 \mathrm{~mm})$ and severe $(\mathrm{CAL}=\geq 5 \mathrm{~mm})$ periodontitis. Psychosocial stress of the individuals was evaluated using occupational stress index. Serum MDA level was evaluated using spectrochromatometer.

Results: Patients with stress demonstrated increased levels of MDA along with a higher loss of attachment.

Conclusion: The results revealed statistically significant association between psychosocial stress and periodontitis indicating psychosocial stress as a risk factor for developing periodontitis.

Keywords: Free radicals, malondialdehyde, oxidative biomarker, periodontitis, psychosocial stress.
\end{abstract}

\section{INTRODUCTION}

The American Academy of Periodontology (AAP) defines periodontitis as an inflammation of the supporting tissues of the teeth characterised by a progressively destructive change leading to loss of bone and periodontal ligament (that is, an extension of inflammation from gingiva into the adjacent bone and periodontal ligament). ${ }^{1}$ Apart from bacterial aetiology, several environmental and genetic factors also modulate periodontal disease progression one of which could be psychosocial stress. ${ }^{2}$

\section{Correspondence:}

Dr. Keshava Abbayya

Department of Periodontology, School of Dental Sciences, Krishna Institute of Medical Sciences, Deemed University, Karad, Maharashtra, India.

email:dr_keshav16@yahoo.co.in

\section{Citation}

Mokashi A, Abbayya K, Varma S, Zope S, Pisal A. Assessment of Psychosocial Stress and its Effect on Periodontal Tissues using Malondialdehyde as Oxidative Stress Biomarker. J Nepal Soc Perio Oral Implantol. 2018;2(2):34-9.
Stress, a term continually being redefined, refers to a psychophysiological response of a living organism to a perspective challenge, change or threat and considered as an important factor in the aetiology and maintenance of many inflammatory diseases. ${ }^{3}$ According to the American Institute of Stress and World Health Organisation, the most common form of stress in the world is occupational stress. Occupational (job) stress is a psychosocial disorder which is an impact of the interaction between the worker and his work environment on the worker. Growing evidence suggests that the psychosocial factors of stress, depression and level of social support provoke changes in host defence mechanisms that modify disease process.

Stress has gained attention in dentistry and psychiatric medicine and is reported to be associated with various diseases. Hence, an effort has been made to correlate psychosocial stress and periodontitis using serum malondialdehyde (MDA) as an oxidative stress biomarker. 


\section{MATERIALS AND METHODS}

The present cross-sectional study was conducted to assess the correlation between psychological stress and periodontitis using MDA as oxidative stress biomarker from July 2016 to February 2017. Ethical clearance was obtained from the Institutional Ethical Committee of the Krishna Institute of Medical Sciences Deemed University, Karad, Maharashtra, India (Reg. No.: KIMSDU/IEC/03/2015). The study included teachers from five different schools situated in Karad, Maharashtra, India. All the teachers were explained about the possible relationship between stress and periodontitis. The study design included evaluation of clinical parameters, psychosocial stress evaluation and also estimation of serum MDA level. A written informed consent was obtained from the participants before carrying out clinical and haematological examination which was in accordance with the World Medical Association's Declaration of Helsinki. ${ }^{4}$

A total of 284 individuals were explained about the study. Among them, 201 individuals between 20 and 60 years of age who fulfilled the criteria of the study and were willing to participate in the study were included. The individuals with minimum of twenty teeth in oral cavity and who have not received any periodontal therapy or not taken antibiotics, anti-inflammatory drugs or any other drugs for at least six months prior to commencement of study were included. Individuals with the history of tobacco in any form, having history of any systemic diseases (cardiovascular diseases, rheumatoid arthritis, diabetes mellitus, renal diseases, etc.), pregnant, lactating and post-menopausal women and patients on vitamin and antioxidant supplements were excluded.

The data was collected by a single examiner (MA) using a standardised proforma which consisted of demographic and personal data clinical periodontal examination, occupational stress evaluation and serum MDA level estimation. Before initiating the clinical examination, the examiner underwent a thorough clinical training at the Dpartment of Periodontology, School of Dental Sciences, Karad, India. The calibration and evaluation of intraexaminer reliability was assessed on 15 randomly selected cases which were measured twice on two different days. The results revealed that the examiner was found to maintain more than $90 \%$ agreement on repeated measures of clinical examination protocol. A careful periodontal examination was carried out and checked for: Probing Pocket Depth (PPD, distance measured from marginal gingiva to the base of the pocket), Clinical Attachment Loss (CAL, distance measured from cementoenamel junction to the base of the pocket/sulcus), Plaque Index (PI by Silness and Löe, 1963) and Gingival Index (GI, by Löe and Silness, 1963). ${ }^{5}$ Each patient was examined using a mouth mirror and University of North Carolina-15 (UNC-15) graduated periodontal probe. After recording the clinical parameters and indices, enrolled individuals were further divided into four groups depending on CAL as described by AAP 1999 classification of periodontal diseases and conditions into healthy, slight ( $\mathrm{CAL}=1-2 \mathrm{~mm})$, moderate $(\mathrm{CAL}=3-4 \mathrm{~mm})$ and severe $(\mathrm{CAL}=\geq 5 \mathrm{~mm})$.

The enrolled individuals were further evaluated for psychosocial stress by using the occupational stress index (OSI, a psychological evaluation tool) put forth by Srivastava and Singh. ${ }^{6}$ The index consisted of a questionnaire which was originally designed in English and it was modified to Hindi language (local language) for a better understanding of the questions by the participants.

The questionnaire consisted of 46 questions which had to be answered by each enrolled study individuals. Each question was rated on a five-point scale. Out of the 46 questions, 28 were "True-keyed" and the rest of the 18, were "False-keyed." The questions in the questionnaire were related to almost all relevant components of the job life which could cause stress in some way or other, such as role over-load, role ambiguity, role conflict, group and political pressures, the responsibility for persons, under-participation, powerlessness, poor peer relationship, intrinsic impoverishment, low status, strenuous working conditions and unprofitability. The summation of the individual scores of all the 46 questions gave the occupational stress index score of each participant.

After periodontal examination and psychosocial stress evaluation, the individuals were assessed for serum MDA level estimation. Blood (5 ml) was drawn from Antecubital vein of forearm using 20 gauge needle and plain vacutainer. The blood sample was centrifuged at $2000 \mathrm{rpm}$ to separate the serum. MDA in serum was separated and determined as conjugate with Thiobarbituric acid (TBA) 0.6\%. Serum proteins were precipitated by Trichloroacetic acid $17.5 \%$ and $70 \%$ and then removed by centrifugation. The MDA-TBA complex was measured at $534 \mathrm{~nm}$ on spectrochromatometer. The concentration of MDA $(\mathrm{nmol} / \mathrm{ml})$ was calculated by using the following formula: Concentration of the test=Absorbent (test)Absorbent (blank)/1.56 x 1000000.

The data which was obtained from the study groups (healthy, slight, moderate and severe periodontitis) were analysed by using ANOVA test (F-test), Tukey's multiple comparison test and Pearson's Correlation. ANOVA compared the means of variables between the four groups. Tukey's multiple comparison test was used to adjust the level of significance in ANOVA. Pearson's correlation measured the correlation between the variables in the different groups.

\section{RESULTS}

The total study population consisted of 201 individuals out of which 111 were female (55\%) and 90 were male (45\%). The mean age of study individuals were $42.08 \pm 6.94$ years. All the groups were homogeneous in their ages and that there was no significant age difference between the groups (Table 1). 
Table 1: Age wise distribution of the study subjects.

\begin{tabular}{|l|l|c|c|c|c|}
\hline \multicolumn{1}{|c|}{ Gender } & $\mathrm{n}(\%)$ & Minimum & Maximum & Mean \pm S.D. \\
\hline \multirow{2}{*}{ Male } & Age & 90 & 26 & 53 & $42.06 \pm 7.017$ \\
\hline \multirow{2}{*}{ Female } & (Years) & $(45)$ & & & \\
\hline
\end{tabular}

Table 2: Mean and standard deviation of periodontal parameters, occupational stress index and serum malondialdehyde level of study subjects.

\begin{tabular}{|l|c|c|c|c|c|c|}
\multicolumn{1}{c|}{ Parameters } & Healthy & Slight & Moderate & Severe & F- value & P-value \\
\hline PPD & $1.66 \pm 0.82$ & $1.68 \pm 0.66$ & $3.41 \pm 1.22$ & $4.95 \pm 1.06$ & 125.79 & $0.00 *$ \\
\hline CAL & $0.00 \pm 0.00$ & $1.37 \pm 0.49$ & $3.46 \pm 0.60$ & $6.33 \pm 0.82$ & 175.39 & $0.00 *$ \\
\hline PI & $0.47 \pm 0.29$ & $0.61 \pm 0.37$ & $1.45 \pm 0.46$ & $2.17 \pm 0.47$ & 182.62 & $0.00 *$ \\
\hline GI & $0.46 \pm 0.30$ & $0.53 \pm 0.38$ & $1.53 \pm 0.50$ & $2.25 \pm 0.48$ & 194.88 & $0.00 *$ \\
\hline OSI & $79.53 \pm 23.62$ & $129.20 \pm 28.97$ & $133.20 \pm 30.46$ & $160.27 \pm 32.39$ & 144.08 & $0.00 *$ \\
\hline MDA & $2.05 \pm 2.63$ & $3.36 \pm 2.49$ & $5.22 \pm 2.52$ & $5.96 \pm 2.33$ & 1.42 & $0.01 *$ \\
\hline
\end{tabular}

ANOVA test was applied to evaluate mean and standard deviation (S.D.) of PPD, CAL, PI, GI, Occupational stress index score and serum MDA levels. The test results revealed that there was a statistically significant difference $(\mathrm{P}<0.001)$ in all the parameters among all groups (healthy, slight, moderate and severe group) (Table 2).

Tukey's post-hoc multiple comparison test also indicated that mean plaque and gingival index score increased with increasing PPD and CAL. Similarly, mean occupational index score and mean serum MDA level increased with increasing CAL (Table 2).

The Pearson's correlation was used to study the association between the occupational stress index score and the clinical attachment level in all four groups. A significant positive correlations were observed between occupational stress index and CAL in moderate and severe periodontitis group $(\mathrm{P}<0.001)$, but not with healthy and slight periodontitis group. Similarly, CAL when correlated with serum MDA level also showed positive significant values in moderate and severe periodontitis group $(\mathrm{P}<0.001)$. Whereas, correlating occupational stress index score and serum MDA level in all the four groups revealed a significant positive association $(\mathrm{P}<0.001)$ between them (Table 3$)$.

\section{DISCUSSION}

Periodontal disease is a bacterially mediated inflammatory disease of the gingiva and adjacent periodontal attachment apparatus. Epidemiologic studies have demonstrated that periodontitis does not affect all individuals in the population in a similar manner. Numerous studies being reviewed indicate that only a subpopulation of $7 \%$ to $15 \%$ of the dentate adult population is affected by advanced destructive periodontal disease. $^{8}$

Apart from bacterial culprit of periodontal destruction certain environmental risk factors also tend to mediate the periodontal disease process such as smoking and diabetes mellitus that modify the host response and thus altering disease progression, severity, and outcome. Other factors, such as stress, depression, and anxiety, are not yet confirmed as absolute risk factors, but have been identified in some observational studies as potential factors that may affect periodontal diseases., ${ }^{9,10}$ The biologic plausibility for stress mediated periodontal destruction is supported by several studies showing that psychosocial conditions, such as depression and exposure to stressor agents, may affect the host immune response, making the individual more

Table 3: Pearson's correlation between clinical attachment level, occupational stress level and serum malondialdehyde level in the study group.

\begin{tabular}{|l|c|c|c|}
\hline \multicolumn{1}{|c|}{ Group } & OSI and CAL & OSI and serum MDA level & CAL and serum MDA Level \\
\hline Healthy & Nil & $0.719 * *$ & Nil \\
\hline Mild & 0.209 & $0.798^{* * *}$ & 0.129 \\
\hline Moderate & $0.489 * *$ & $0.848^{* *}$ & $0.529 * *$ \\
\hline Severe & $0.585^{* *}$ & $0.883^{* *}$ & $0.542^{* * *}$ \\
\hline OSI & $79.53 \pm 23.62$ & $129.20 \pm 28.97$ & $133.20 \pm 30.46$ \\
\hline MDA & $2.05 \pm 2.63$ & $3.36 \pm 2.49$ & $5.22 \pm 2.52$ \\
\hline
\end{tabular}


susceptible to the development of unhealthy conditions and affecting periodontal health. ${ }^{11}$

Stress can be viewed as a process with both psychological and physiological components affecting the periodontium directly or indirectly. The indirect route involves the psychological aspect of a person with health impairing behaviour like poor oral hygiene, smoking, alcohol consumption and poor nutritional intake. The direct route involves the alteration of the resistance of the periodontium to infection. ${ }^{12}$ Several studies show that psychosocial stress can down-regulate the cellular immune response which can be explained by three hypothesis, (i) monocyte T-lymphocyte hypothesis of depression, (ii) cytokine hypothesis of depression and (iii) inflammation and oxidative/ nitrosative stress hypothesis of depression. The cascade of events in monocyte T-lymphocyte and cytokine hypothesis are interlinked. This interrelation between immune cells and cytokine begins with the stimulation of Hypothalamus-Pituitary Axis which plays a key role in stress response and can serve as a prototype for coordination of psychosocial information into physiological response and immune modulation. ${ }^{13}$

In oxidative and nitrosative stress hypothesis of depression there is decrease in antioxidant level such as vitamin E, omega-3 fatty acid in stressful conditions. This will progressively lead to increase in reactive oxygen species (ROS) and reactive nitrogen species (RNS) collectively called as free radical species. Excessive free radicals further can effectuate the disease process by causing damage to proteins, carbohydrate, fatty acids, deoxyribonucleic acid (DNA) etc. Damage to the free fatty acid generates xanthine peroxidase which leads to increase in lipid peroxidation. Polyunsaturated fatty acids are more vulnerable to lipid peroxidation which is evident by increase in serum MDA level as advanced lipoxidation end product (ALE). ${ }^{14}$ MDA targets the mitochondria causing mitochondrial damage by depressing mitochondrial Glutathione (GSH) and Superoxide dismutase (SOD) concentration, increases mitochondrial ROS and protein carbonyls and inhibits mitochondrial respiratory process thus causing degenerative changes in the tissue. This oxidative stress hypothesis explains the link between degenerative changes associated with destructive periodontal diseases and psychosocial stress. ${ }^{15}$

In the current study association between psychosocial stress and periodontitis was explained by evaluating serum MDA as indicator/biomarker for oxidative stress. The study revealed a significant difference for PPD and CAL in all four groups and all study groups also showed significant differences in mean occupational stress index score which indicate that severity of periodontal disease increases with increase in psychosocial stress. These findings are in agreement with the literature which revealed that depression was associated with a more rigorous course of periodontitis. ${ }^{10}$
Studies have revealed significantly higher self-reported depression and loneliness in chronic periodontitis and generalised aggressive periodontitis than in control group when examined for psychosocial variables. ${ }^{12}$ Systematic review analysis has considered psychosocial stress as a potential risk factor for periodontal disease. ${ }^{16}$ A study conducted by Moss et al correlated various parameters of periodontal diseases with measures of psychological stress, distress and coping behaviours and reported that the effects of stress on periodontal diseases can be moderated by adequate coping behaviours. They further concluded that psychosocial measures of stress associated with strain and distress are significant risk indicators for severe periodontal disease in

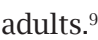

Correlation between psychosocial stress and periodontitis in present study showed moderate positive correlation in moderate $(r=0.489)$ and severe periodontitis $(r=0.585)$ groups ( $\mathrm{P}=<0.01$ ) indicating that psychosocial stress can be considered as one of the relative but not an absolute risk indicator for periodontitis. A cross-sectional study evaluated the prevalence of negative life events and psychological factors and their relation to periodontal disease, and concluded that traumatic life events such as the loss of a spouse may increase the risk for periodontal disease. ${ }^{17}$ An individual's coping behaviour may play a role in the progression of periodontal disease. The patients with periodontitis and inadequate stress-coping strategies are at greater risk for severe periodontal diseases when compared to periodontally healthy individuals. ${ }^{18}$ Deinzer et al conducted a case-control study to evaluate whether a range of life-events were associated with an objective measure of periodontitis in adults and concluded that psychosocial factors and oral health behaviours cluster together as important determinants of periodontitis. ${ }^{19}$ It is obvious that proper oral hygiene is partially dependent on the mental health status of the patient. It has also been reported that psychological disturbances can lead patients to neglect oral hygiene and that the resultant accumulation of plaque is detrimental to the periodontal tissue.

In the present study, the healthy group had a lower mean plaque score and gingival index score as compared to the mild, moderate and severe groups. Further, the mild group had a lower mean plaque score as compared to the moderate and severe periodontitis groups. These findings were in accordance with the study which reported increased dental plaque accumulation and gingival inflammation in medical students who were under academic stress. ${ }^{20,21}$ Emotional conditions are thought to modify dietary intake, thus indirectly affecting periodontal status. Psychological factors affect the choice of foods, the physical consistency of the diet, and the quantities of food eaten. This can involve, for instance, the consumption of excessive quantities of refined carbohydrates and softer diets requiring less vigorous mastication and 
therefore predisposing to plaque accumulation at the risk site. In contrast to the above statement, relationship between stress and anxiety with chronic periodontitis was evaluated concluding that there is no correlation between mean plaque score and perceived life events and related stress score..$^{22}$

Psychosocial stress is said to be associated with the increased oxidative burst in the body which further lead to tissue destruction. Serum MDA level which is the end product of lipid peroxidation of ROS pathway is found to be significantly increased in major depression. ${ }^{23}$ Recent data suggest increased oxidative stresses and MDA play a key role in many chronic diseases such as periodontitis, atherosclerosis, neurodegenerative disorders, etc. ${ }^{24,25}$ In the present study, the result revealed that the mean serum MDA level increased with increase in CAL which was different in all four groups ( $\mathrm{P}=$ $<0.01)$. Furthermore, Pearson's correlation showed significant positive association between mean serum MDA level and mean CAL in moderate and severe periodontitis group and no significant correlation was observed in healthy group and individuals with mild periodontitis. Consistent with the results of current study a significant increase in MDA level in the periodontitis group was demonstrated by Celec et al. ${ }^{25}$ Similarly, higher serum MDA level were found in periodontitis and that oxidative stress is thought to be a predisposing factor for MDA production in periodontitis. ${ }^{26}$
A significant elevation of MDA and reductions in antioxidant enzymes was reported in patients with periodontitis and also a direct correlation between MDA levels and an inverse correlation of antioxidant enzymes in periodontitis was observed. Our results are consistent with studies demonstrating an increase in lipid peroxidation levels in serum of individuals with periodontitis. ${ }^{27}$

Thus, present study supports the positive correlation between psychosocial stress and periodontitis. Psychosocial stress along with the other factors such as age, socio-economic condition can be considered as one of the risk indicator to develop periodontal disease.

\section{CONCLUSION}

The current study concluded with a positive association between periodontitis and psychosocial stress. But, for a better understanding of this possible relationship, studies involving larger sample size and also taking into consideration the demographic data such as socioeconomic status and age has to be given priority. It is equally important to take into account that more longitudinal study designs are required to prove the association.

\section{REFERENCES}

1. Armitage GC. Development of a classification system for periodontal diseases and conditions. Ann Periodontol. 1999;4(1):1-6.

2. Loesche WJ, Grossman NS. Periodontal disease as a specific, albeit chronic, infection: diagnosis and treatment. Clin Microbiol Rev. 2001;14:727-52.

3. Dhabhar FS. A hassle a day may keep the pathogens away: the fight-or-flight stress response and the augmentation of immune function. Integr Comp Biol. 2009;49(3):215-36.

4. Löe H. The gingival index, the plaque index and the retention index systems. The J Periodontol. 1967;38(6P2):610-6.

5. Srivastava AK, Singh AP. Manual of the occupational stress index. Varanasi, UP: Manovaigyanik Parikcchan Sansthan;1981.

6. Bhutia Y, Ghosh A, Sherpa ML, Pal R, Mohanta PK. Serum malondialdehyde level: Surrogate stress marker in the Sikkimesediabetics. J Nat Sci Biol Med. 2011;(1):107-12.

7. Johnson NW, Griffiths GS, Wilton JM, Maiden MF, Curtis MA, Gillett IR, Wilson DT, Sterne JA. Detection of high-risk groups and individuals for periodontal diseases: Evidence for the existence of high-risk groups and individuals and approaches to their detection. J Clin Periodontol. 1988;15(5):276-82

8. Genco RJ. Current view of risk factors for periodontal diseases. J Periodontol. 1996;67(10s):1041-9.

9. Moss ME, Beck JD, Kaplan BH, Offenbacher S, Weintraub JA, Koch GG, et al. Exploratory caseDcontrol analysis of psychosocial factors and adult periodontitis. J Periodontol. 1996;67:1060-9.

10. Irwin M, Patterson T, Smith TL, Caldwell C, Brown SA, Gillin JC, et al. Reduction of immune function in life stress and depression. Biol Psychiatry. 1990;27(1):22-30.

11. Page RC, Offenbacher S, Schroeder HE, Seymour GJ, Kornman KS. Advances in the pathogenesis of periodontitis: summary of developments, clinical implications and future directions. Periodontol 2000. 1997;14(1):216-48.

12. Monteiro da Silva AM, Oakley DA, Newman HN, Nohl FS, Lloyd HM. Psychosocial factors and adult onset rapidly progressive periodontitis. J Clin Periodontol. 1996;23:789-94.

13. Breivik T, Thrane PS, Murison R, Gjermo P. Emotional stress effects on immunity, gingivitis and periodontitis. Eur J Oral Sci. 1996;104(4):327-34.

14. Maes M, Yirmyia R, Noraberg J, Brene S, Hibbeln J, Perini G, et al. The inflammatory \& neurodegenerative (I\&ND) hypothesis of depression: leads for future research and new drug developments in depression. Metab Brain Dis. 2009;24(1):27-53.

15. Del Rio D, Stewart AJ, Pellegrini N. A review of recent studies on malondialdehyde as toxic molecule and biological marker of oxidative stress. Nutr Metab Cardiovasc Dis. 2005;15(4):316-28.

16. Hugoson A, Ljungquist B, Breivik T. The relationship of some negative events and psychological factors to periodontal disease in an adult Swedish population 50 to 80 years of age. J Clin Periodontol. 2002;29(3):247-53. 
17. Wimmer G, Janda M, Wieselmann-Penkner K, Jakse N, Polansky R, Pertl C. Coping with stress: its influence on periodontal disease. J Periodontol. 2002;73(11):1343-51.

18. Croucher R, Marcenes WS, Torres MC, Hughes F, Sheiham A. The relationship between life-events and periodontitis A case-control study. J Clin Periodontol. 1997;24(1):39-43.

19. Deinzer R, Förster P, Fuck L, Herforth A, Stiller-Winkler R, Idel H. Increase of crevicular interleukin $1 \mathrm{~b}$ under academic stress at experimental gingivitis sites and at sites of perfect oral hygiene. J Clin Periodontol. 1999;26(1):1-8.

20. Kurer JR, Watts TL, Weinman J, Gower DB. Psychological mood of regular dental attenders in relation to oral hygiene behaviour and gingival health. J Clin Periodontol. 1995;22(1):52-5.

21. Khanzode SD, Dakhale GN, Khanzode SS, Saoji A, Palasodkar R. Oxidative damage and major depression: the potential antioxidant action of selective serotonin re-uptake inhibitors. Redox Rep. 2003;8(6):365-70.

22. Vettore MV, Leão AT, Monteiro Da Silva AM, Quintanilha RS, Lamarca GA. The relationship of stress and anxiety with chronic periodontitis. J Clin Periodontol. 2003;30(5):394-402.

23. Almerich-Silla JM, Pastor S, Serrano F, Puig-Silla M, Dasí F. Oxidative stress parameters in saliva and its association with periodontal disease and types of bacteria. Dis Markers. 2015;2015:653537.

24. Halliwell B, Whiteman M. Measuring reactive species and oxidative damage in vivo and in cell culture: how should you do it and what do the results mean? Br J Pharmacol. 2004;142(2):231-55.

25. Celec P, Hodosy J, Celecová V, Vodráźka J, Červenka T, Halčák L, et al. Salivary thiobarbituric acid reacting substances and malondialdehyde-their relationship to reported smoking and to periodontal status described by the papillary bleeding index. DisMarkers. 2005;21(3):133-7.

26. Mashayekhi F, Aghahoseini F, Rezaie A, Zamani MJ, Khorasani R, Abdollahi M. Alteration of cyclic nucleotides levels and oxidative stress in saliva of human subjects with periodontitis. J Contemp Dent Pract. 2005;6(4):46-53.

27. Maxwell SR, Dietrich T, Chapple IL. Prediction of serum total antioxidant activity from the concentration of individual serum antioxidants. Clinic Chim Acta. 2006 Oct;372(1-2):188-94. 